\title{
An Extreme Point Result for Robust Stability of a Diamond of Polynomials
}

\section{B. R. Barmish; R. Tempo † C. V. Hollot $\ddagger$ and H. I. Kang*}

\author{
*Department of Electrical and \\ Computer Engineering, University of \\ Wisconsin-Madison, Madison, \\ Wisconsin $\mathbf{5 3 7 0 6}$
}

\author{
${ }^{\dagger}$ CENS-CNR, Politecnico di Torino, \\ Corso Duca degli Abruzzi 24, 10129 Torino \\ Italy
}

\author{
$\ddagger$ Department of Electrical and \\ Computer Engineering, University of \\ Massachusetts, Amherst \\ Massachusetts 01003
}

\section{Abstract}

The main result of this paper is that a family of polynomials with real coefficients lying in a diamond is Hurwitz if and only if eight distinguished extreme polynomials are Hurwitz. For the case of complex coefficients, it is shown via counterexample that no analogous extreme point result holds.

\section{Introduction}

Motivated by Kharitonov's seminal theorem [1] on robust stability for a box of polynomials, a number of recent papers have concentrated on the so-called dual problem for a diamond of polynomials; see Tempo [2] [3], Bose and Kim [4] and Katbab and Jury [5]. This robust stability problem for diamonds is simply stated as follows: Consider an $n$-th order polynomial $p(s, q)=q_{0}+q_{1} s+q_{2} s^{2}+\ldots$ $+q_{n-1} s^{n-1}+q_{n} s^{n}$ with real coefficients $q_{i}$ known to lie in the $(n+1)$-dimensional diamond with center $q^{*}=\left(q_{0}^{*}, q_{1}^{*}, \cdots, q_{n}^{*}\right)$ and radius $r>0$; i.e., admissible coefficients are described by $q \in Q_{r}$ where $q=\left(q_{0}, q_{1}, \cdots, q_{n}\right)$ and

$Q_{r} \doteq\left\{q:\left|q_{0}-q_{0}^{*}\right|+\left|q_{1}-q_{1}^{*}\right|+\ldots+\left|q_{n}-q_{n}^{*}\right| \leq r\right\}$.

In order to guarantee that $p(s, q)$ has degree $n$ for all $q \in Q_{r}$, it is assumed that $\left|q_{n}^{*}\right|>r$.

Now, we denote the diamond family by $\mathcal{P} \doteq\left\{p(\cdot, q): q \in Q_{r}\right\}$ and say that $\mathcal{P}$ is Hurwitz if $p(s, q)$ has all its roots in the open left half plane for all $q \in Q_{r}$. In [2] and [3], it is shown that $\mathcal{P}$ is Hurwitz if and only if eight distinguished exposed edges of $\mathcal{P}$ are Hurwitz; note that the number "eight" is independent of the order $n$. In [4] and [5], analogous edge results are provided for the case of complex coefficients and unit circle stability.

The main goal of this paper is to prove that there is no need to check stability of edges-it is necessary and sufficient to check if eight distinguished extreme polynomials are Hurwitz. As discussed in the conclusion of this paper, it is also important to note that an analogous extreme point result does not hold for diamonds of complex polynomials. This is demonstrated with an example for which $\mathcal{P}$ is not Hurwitz but all extreme polynomials in $\mathcal{P}$ are Hurwitz.

\section{The Main Result}

The main result of this paper is easy to describe.

Theorem: The diamond family of polynomials $\mathcal{P}$ is Hurwitz if and only if the eight extreme polynomials

$p_{1}(s) \doteq p\left(s, q^{*}\right)+r ; p_{2}(s) \doteq p\left(s, q^{*}\right)-r ;$

$p_{3}(s) \doteq p\left(s, q^{*}\right)+r s ; p_{4}(s) \doteq p\left(s, q^{*}\right)-r s ;$

$p_{5}(s) \doteq p\left(s, q^{*}\right)+r s^{n-1} ; p_{6}(s) \doteq p\left(s, q^{*}\right)-r s^{n-1} ;$

are Hurwitz.

$p_{7}(s) \doteq p\left(s, q^{*}\right)+r s^{n} ; p_{8}(s) \doteq p\left(s, q^{*}\right)-r s^{n}$

Remark: In some cases, it is of interest to deal with diamond family with the added restriction that perturbations are "one sided." By this, we mean that for all $i, q_{i} \geq q_{i}^{*}$. In this case we obtain a so-called simplex polynomial family and by using a line of proof quite similar to that used for the theorem, it can be shown that this family is Hurwitz if and only if the five extreme polynomials $v_{1}(s) \doteq p\left(s, q^{*}\right) ; v_{2}(s) \doteq p\left(s, q^{*}\right)+r ; v_{3}(s) \doteq p\left(s, q^{*}\right)+r s ;$ $v_{4}(s) \doteq p\left(s, q^{*}\right)+r s^{n-1} ; v_{5}(s) \doteq p\left(s, q^{*}\right)+r s^{n}$ are Hurwitz.

\section{Proof of the Main Result}

To prove the main result, three fundamental lemmas will be used. The first lemma is the edge result which was discussed in the introduction.

Lemma 1: (see [3] for proof): The diamond family of polynomials $\mathcal{P}$ is Hurwitz if and only if the eight polynomials

$$
\begin{aligned}
& e_{1}(s, \lambda)=p\left(s, q^{*}\right)-\lambda r-(1-\lambda) r s ; \\
& e_{2}(s, \lambda)=p\left(s, q^{*}\right)+\lambda r-(1-\lambda) r s ; \\
& e_{3}(s, \lambda)=p\left(s, q^{*}\right)+\lambda r+(1-\lambda) r s ; \\
& e_{4}(s, \lambda)=p\left(s, q^{*}\right)-\lambda r+(1-\lambda) r s ; \\
& e_{5}(s, \lambda)=p\left(s, q^{*}\right)-\lambda r s^{n-1}-(1-\lambda) r s^{n} ; \\
& e_{6}(s, \lambda)=p\left(s, q^{*}\right)+\lambda r s^{n-1}-(1-\lambda) r s^{n} ; \\
& e_{7}(s, \lambda)=p\left(s, q^{*}\right)+\lambda r s^{n-1}+(1-\lambda) r s^{n} ; \\
& e_{8}(s, \lambda)=p\left(s, q^{*}\right)-\lambda r s^{n-1}+(1-\lambda) r s^{n}
\end{aligned}
$$

are Hurwitz for all $\lambda \in[0,1]$.

The next lemma is a known result relating real and complex Hurwitz polynomials.

Lemma 2: (see pp. 61 of [6] for proof): Consider a real coefficient polynomial $p(s)$ expressed as $p(s)=f\left(s^{2}\right)+s g\left(s^{2}\right)$ where $f(\cdot)$ and $g(\cdot)$ are also polynomials and assume that $p(s)$ has positive coefficients. Then $p(s)$ is Hurwitz if and only if the complex coefficient polynomials $\tilde{p}_{1}(s)=f(j s)+j g(j s)$ and $\tilde{p}_{2}(s)=f(-j s)+s g(-j s)$ are Hurwitz.

The next lemma is a minor extension of Lemma 2.1 in Hollot and Yang [7]; see also the forthcoming paper by Hollot, Kraus, Tempo and Barmish [8] where a different extension is given. For the reader interested in the proof, it is worth noting that the proof of Lemma 3 below involves a geometric argument whereas the original proof in [7] is algebraic in nature; in [7], the Lienard-Chipart criterion is used.

Lemma 3: Let $p_{0}(s)$ be a fixed polynomial with real coefficients. In addition, take $r>0, \alpha \in\{-1,1\}, \beta \in\{-1,1\}$ and integer $i \in\{0, n-1\}$ and assume that the polynomial

$$
p(s, \lambda)=p_{0}(s)+\lambda r s^{i}(\alpha+\beta s)
$$

has degree $n$ for all $\lambda \in[0,1]$. Then $p(s, \lambda)$ is Hurwitz for all $\lambda \in[0,1]$ if and only if $p(s, 0)$ and $p(s, 1)$ are Hurwitz.

Proof: Since necessity is obvious, we proceed to establish sufficiency. We consider the case when $\alpha=\beta=1, n$ is odd and $i=n-1$ and simply note that a nearly identical proof applies for the other cases; e.g., if $i=n-1$ and $n$ is even, then in using Lemma 2 one exploits $\tilde{p}_{2}(s)$ rather than $\tilde{p}_{1}(s)$ in the argument below.

Indeed, we assume that $p(s, 0)$ and $p(s, 1)$ are Hurwitz. Hence, each of these two polynomials has either all positive coefficients or all negative coefficients. We claim that both polynomials must have coefficients of the same sign. To establish this claim, we proceed by contradiction; i.e., suppose $p(s, 0)$ and $p(s, 1)$ have coefficients of opposite sign. Then letting $a_{n}$ denote the coefficient of $s^{n}$ in $p(s, 0)=p_{0}(s)$, it follows that the coefficient of $s^{n}$ in $p(s, \lambda)$ is $a_{n}(\lambda)=a_{n}+\lambda r$. Notice that if $a_{n}(0)$ and $a_{n}(1)$ have opposite sign, it follows that $a_{n}\left(\lambda^{*}\right)=0$ for some $\lambda^{*} \in(0,1)$. This contradicts the assumption that $p(s, \lambda)$ has degree $n$ for all $\lambda \in[0,1]$. Henceforth, 
without loss of generality, we assume that both $p(s, 0)$ and $p(s, 1)$ have positive coefficients. As an immediate consequence, it follows that $p(s, \lambda)$ has positive coefficients for all $\lambda \in[0,1]$.

The next step of the proof is to write $p_{0}(s)=f_{0}\left(s^{2}\right)+s g_{0}\left(s^{2}\right)$ where $f_{0}(\cdot)$ and $g_{0}(\cdot)$ are polynomials. This leads to

$$
p(s, \lambda)=\left[f_{0}\left(s^{2}\right)+\lambda r s^{n-1}\right]+s\left[g_{0}\left(s^{2}\right)+\lambda r s^{n-1}\right] .
$$

Now, in accordance with Lemma 2 , it suffices to show that the complex coefficient polynomial

$$
\begin{aligned}
\tilde{p}(s, \lambda) & =\left[f_{0}(j s)+\lambda r(j s)^{(n-1) / 2}\right]+j\left[g_{0}(j s)+\lambda r(j s)^{(n-1) / 2}\right] \\
& =\left[f_{0}(j s)+j g_{0}(j s)\right]+\lambda r(j s)^{(n-1) / 2}(1+j)
\end{aligned}
$$

is Hurwitz for all $\lambda \in[0,1]$. To this end, we first note that we can apply Lemma 2 to the extremes; i.e., Hurwitzness of $p(s, 0)$ and $p(s, 1)$ implies that $\tilde{p}(s, 0)$ and $\tilde{p}(s, 1)$ are Hurwitz. Now, to establish that $\tilde{p}(s, \lambda)$ is Hurwitz for all $\lambda \in[0,1]$, we proceed by contradiction: If $\tilde{p}(s, \tilde{\lambda})$ is not Hurwitz for some $\tilde{\lambda} \in(0,1)$, then continuous root dependence on $\lambda$ dictates that there exists some $\omega^{*} \in \mathbf{R}$ and $\lambda^{*} \in(0,1)$ such that

$$
\tilde{p}\left(j \omega^{*}, \lambda^{*}\right)=0 \text {. }
$$

We first rule out the possibility that $\tilde{p}\left(j \omega^{*}, 0\right)=0$ or $\tilde{p}\left(j \omega^{*}, 1\right)=0$ or $\omega^{*}=0$ because this would contradict Hurwitzness of $\tilde{p}(s, 0)$ and $\tilde{p}(s, 1)$. Furthermore, noting that the quantity multiplying $\lambda$ in (4) is nonzero except at $\omega=0$, it follows that for each $\omega \neq 0$, the value set $\tilde{p}(j \omega,[0,1]) \doteq\{\tilde{p}(j \omega, \lambda): \lambda \in[0,1]\}$ is a line segment in the complex plane with endpoints $\tilde{p}(j \omega, 0)$ and $\tilde{p}(j \omega, 1)$ and constant slope $\tilde{m}(\omega) \equiv 1$.

To complete the proof, note that (5) implies that the point $s=0$ lies on the relative interior of the line segment $\tilde{p}\left(j \omega^{*},[0,1]\right)$. Since the polynomials $\tilde{p}(s, 0)$ and $\tilde{p}(s, 1)$ are Hurwitz, their angles $\Delta \tilde{p}(j \omega, 0)$ and $\Delta \tilde{p}(j \omega, 1)$ are strictly increasing functions of $\omega$. Using this fact in conjunction with the fact that $s=0$ lies on $p\left(j \omega^{*},[0,1]\right)$, it follows that for $\omega>\omega^{*}$ with $\left|\omega-\omega^{*}\right|$ sufficiently small, the value set has slope $\tilde{m}(\omega)>\tilde{m}\left(\omega^{*}\right)=1$. This, however, contradicts the constancy of $\tilde{m}(\omega)$. The proof of lemma is now complete. $\square$

Remark: Note that the restrictions on $i, \alpha$ and $\beta$ are stronger than necessary in order for the conclusion of Lemma 3 to hold; the more restrictive version of the lemma is all that we will require to prove the main theorem. By examining the proof, however, it is quite easy to verify that a stronger version of the lemma holds under the conditions that $\alpha$ and $\beta$ are real and $i \in\{0,1,2,3, \cdots$, $n-2, n-1\}$.

Now, we are in a position to prove the main result.

Proof of Theorem: First, note that necessity is trivial since each $p_{k}(s)$ is a member of $\mathcal{P}$. To establish sufficiency, we first invoke Lemma 1 ; i.e., the diamond family of polynomials $\mathcal{P}$ is Hurwitz if and only if the eight edges given in (2) are Hurwitz. Next, observe that by substitution of $\lambda=0$ and $\lambda=1$ into the $e_{k}(s, \lambda)$, we obtain precisely the eight polynomials $p_{1}(s), p_{2}(s), \cdots, p_{8}(s)$. Hence, the remainder of the proof reduces to showing that for $k \in\{1,2,3, \cdots, 8\}$, $e_{k}(s, 0)$ and $e_{k}(s, 1)$ being Hurwitz implies that $e_{k}(s, \lambda)$ is Hurwitz for all $\lambda \in[0,1]$. This is easily accomplished by verifying that each $e_{k}(s, \lambda)$ satisfies the preconditions of Lemma 3 . That is, for arbitrary $k \in\{1,2,3, \cdots, 8\}, e_{k}(s, \lambda)$ can be written in the form (3), $e_{k}(s, \lambda)$ has degree $n$ and $e_{k}(s, 0)$ and $e_{k}(s, 1)$ are Hurwitz.

\section{Example}

Consider the diamond family $\mathcal{P}$ of the fourth order polynomials described by $p(s, q)=q_{0}+q_{1} s+q_{2} s^{2}+q_{3} s^{3}+q_{4} s^{4}$ with real coefficients $q_{i}$ known to lie in the diamond with center

$q^{*}=\left(q_{0}^{*}, q_{1}^{*}, q_{2}^{*}, q_{3}^{*}, q_{4}^{*}\right)=(3.4900,7.9800,6.4900,3.0000,1.0000)$ and radius $r=0.5$. Since $q_{4}^{*}>r$, it is immediate that all polynomials in this family are fourth order. Therefore, by the main theorem, Hurwitzness of the eight extreme polynomials

$$
\begin{aligned}
& p_{1}(s)=p\left(s, q^{*}\right)+0.5 ; p_{2}(s)=p\left(s, q^{*}\right)-0.5 ; \\
& p_{3}(s)=p\left(s, q^{*}\right)+0.5 s ; p_{4}(s)=p\left(s, q^{*}\right)-0.5 s ;
\end{aligned}
$$

$$
\begin{aligned}
& p_{5}(s)=p\left(s, q^{*}\right)+0.5 s^{3} ; p_{6}(s)=p\left(s, q^{*}\right)-0.5 s^{3} ; \\
& p_{7}(s)=p\left(s, q^{*}\right)+0.5 s^{4} ; p_{8}(s)=p\left(s, q^{*}\right)-0.5 s^{4}
\end{aligned}
$$

is neccesary and sufficient for $\mathcal{P}$ to be Hurwitz. Now, it is straightforward to verify that this is indeed the case; verify that each $p_{k}(s)$ has all its roots in the open left half plane.

For this same example, we now compute a measure of robustness. That is, we compute the largest value of the radius $r$ for which Hurwitzness is preserved. Calling this robustness measure $r_{\max }$, we already know from the analysis above that $r_{\max }>0.5$.

Now, for each $p_{k}(s)$, let $r_{\max , k}$ denote the largest value of $r$ such that $p_{k}(s)$ is Hurwitz. A straightforward computation leads to $r_{\max , 1} \approx 6.6978 ; r_{\max , 2}=3.4900 ; r_{\max , 3} \approx 9.7150 ; r_{\max , 4} \approx 6.2050$ $r_{\max , 5} \approx 10.4867 ; r_{\max , 6} \approx 1.6471 ; r_{\max , 7} \approx 0.9467 r_{\max , 8}=1.0000$ from which it follows that $r_{\max }=\min _{k} r_{\max , k} \approx 0.9467$.

\section{Concluding Remarks}

In view of the main result in the paper, there is a temptation to conjecture that an analogous extreme point result can be given for complex polynomials; i.e, instead of $p(s, q)$, consider a polynomial of the form $p(s, u, v)=\sum_{i=0}^{n}\left(u_{i}+j v_{i}\right) s^{i}$ where $u=\left(u_{0}, u_{1}, \cdots, u_{n}\right)$ and $v=\left(v_{0}, v_{1}, \cdots, v_{n}\right)$ lie in diamonds $U$ and $V$ analogous to (1) respectively. It is surprising to note, however, that such a conjecture is false. To this end, the following counterexample is given: Consider a second order complex polynomial $p(s, u, v)$ with $u=\left(u_{0}, u_{1}, u_{2}\right)$, $v=\left(v_{0}, v_{1}, v_{2}\right)$, diamond centers given by $u_{0}^{*}=-4.3176$,

$u_{1}^{*}=0.0111, u_{2}^{*}=1.2272, v_{0}^{*}=1.8398, v_{1}^{*}=15.1285, v_{2}^{*}=6.3118$ and both real and imaginary radii $r_{u}=r_{v}=1$. It is then straightforward to verify that all polynomials in the family are second order and moreover, there are at most 36 extreme polynomials of the form $p\left(s, u^{*}, v^{*}\right) \pm(1 \pm j) ; p\left(s, u^{*}, v^{*}\right) \pm(1 \pm j s) ; p\left(s, u^{*}, v^{*}\right) \pm\left(1 \pm j s^{2}\right)$; $p\left(s, u^{*}, v^{*}\right) \pm(s \pm j) ; p\left(s, u^{*}, v^{*}\right) \pm(s \pm j s) ; p\left(s, u^{*}, v^{*}\right) \pm\left(s \pm j s^{2}\right)$; $p\left(s, u^{*}, v^{*}\right) \pm\left(s^{2} \pm j\right) ; p\left(s, u^{*}, v^{*}\right) \pm\left(s^{2} \pm j s\right) ; p\left(s, u^{*}, v^{*}\right) \bumpeq\left(s^{2} \pm j s^{2}\right)$

which are all Hurwitz. However, the polynomial

$p(s)=p\left(s, u^{*}, v^{*}\right)+0.5 s-(0.5+j)$ is a member of the family and has roots $s_{1} \approx-2.3249-j 0.0440$ and $s_{2} \approx 0.0002-j 0.3271$. Hence the family is not Hurwitz even though all the extremes are Hurwitz.

\section{References}

[1] V. L. Kharitonov, "Asymptotic Stability of an Equilibrium Position of a Family of Systems of Linear Differential Equations," Differential'nye Uraveniya, Vol. 14, no. 11 , pp. $1483-1485,1978$.

[2] R. Tempo, "The Dual Theorem of Kharitonov for a Class of Dependent Uncertainties," Proceedings of the 27th IEEE Conference on Decision and Control, Austin, 1988.

[3] R. Tempo, "A Dual Result to Kharitonov's Theorem," IEEE Transactions on Automatic Control, Vol. AC-34, March 1990.

[4] N. K. Bose and K. D. Kim, "Stability of a Complex Polynomial Set with Coefficients in a Diamond and Generalizations," IEEE Transactions on Circuits and Systems, Vol. 36, pp. 1168-1174, 1989.

[5] A. Katbab and E. I. Jury, "Robust Schur Stability of a ComplexCoefficient Polynomial Set with Coefficients in a Diamond," to appear.

[6] E. I. Jury, Inners and Stability of Dynamic Systems, Wiley, New York, 1974.

7] C. V. Hollot and F. Yang, "Robust Stabilization of Interval Plants using Lead or Lag Compensators," Proceedings of the 28th IEEE Conference on Decision and Control, Tampa, 1989.

[8] C. V. Hollot, F. J. Kraus, R. Tempo and B. R. Barmish, "Extreme Point Results for Robust Stabilization of Interval Plants with First Order Compensators," to appear in the Proceedings of the American Control Conference, San Diego, 1990. 